\title{
Comorbid infections induce progression of visceral leishmaniasis
}

\author{
Angela J. Toepp ${ }^{1,2}$, Glória R. G. Monteiro ${ }^{3}$, José F. V. Coutinho ${ }^{3}$, Adam Leal Lima ${ }^{1,2}$, Mandy Larson ${ }^{1,2}$, \\ Geneva Wilson 1,2, Tara Grinnage-Pulley ${ }^{1,2}$, Carolyne Bennett ${ }^{1,2}$, Kurayi Mahachi ${ }^{1,2}$, Bryan Anderson ${ }^{1,2}$, \\ Marie V. Ozanne, Michael Anderson ${ }^{1,2}$, Hailie Fowler ${ }^{1,5}$, Molly Parrish ${ }^{1,2}$, Kelsey Willardson ${ }^{1,2}$, Jill Saucier ${ }^{6}$, \\ Phyllis Tyrell ${ }^{6}$, Zachary Palmer ${ }^{7}$, Jesse Buch ${ }^{6}$, Ramaswamy Chandrashekar ${ }^{6}$, Grant D. Brown ${ }^{4}$, Jacob J. Oleson ${ }^{4}$, \\ Selma M. B. Jeronimo ${ }^{3}$ and Christine A. Petersen ${ }^{1,2,5^{*}}$ (D)
}

\begin{abstract}
Background: Visceral leishmaniasis (VL) is a vector borne zoonotic disease endemic in humans and dogs in Brazil. Due to the increased risk of human infection secondary to the presence of infected dogs, public health measures in Brazil mandate testing and culling of infected dogs. Despite this important relationship between human and canine infection, little is known about what makes the dog reservoir progress to clinical illness, significantly tied to infectiousness to sand flies. Dogs in endemic areas of Brazil are exposed to many tick-borne pathogens, which are likely to alter the immune environment and thus control of $L$. infantum.

Results: A cross-sectional study of 223 dogs from an area of Natal, in the Rio Grande do Norte, Brazil, were studied to determine the association between comorbid tick-borne disease and Leishmania infection in this endemic area. The risk of Leishmania seropositivity was 1.68x greater in dogs with tick-borne disease seropositivity compared to those without (Adjusted RR: 1.68, 95\% Cl: 1.09-2.61, $P=0.019$ ). A longitudinal study of 214 hunting dogs in the USA was conducted to determine the causal relationship between infection with tick-borne diseases and progression of VL. Hunting dogs were evaluated three times across a full tick season to detect incident infection with tick-borne diseases. A logistic regression model with generalized estimating equations to estimate the parameters was used to determine how exposure to tick-borne disease altered $V L$ progression over these three time points when controlling for other variables. Dogs infected with three or more tick-borne diseases were $11 \times$ more likely to be associated with progression to clinical VL than dogs with no tick-borne disease (Adjusted RR: 11.64, 95\% Cl: 1.22-110.99, $P=0.03$ ). Dogs with exposure to both Leishmania spp. and tick-borne diseases were five times more likely to die during the study period (RR: $4.85,95 \% \mathrm{Cl}: 1.65-14.24, P=0.0051$ ).

Conclusions: Comorbid tick-borne diseases dramatically increased the likelihood that a dog had clinical L. infantum infection, making them more likely to transmit infection to sand flies and people. As an important consequence, reduction of tick-borne disease exposure through topical or oral insecticides may be an important way to reduce progression and transmissibility of Leishmania infection from the canine reservoir to people.
\end{abstract}

Keywords: Tick-borne diseases, Canine leishmaniosis, Risk-factor, Progression

\footnotetext{
* Correspondence: christine-petersen@uiowa.edu

'Department of Epidemiology, College of Public Health, University of lowa, lowa City, lowa 52242, USA

${ }^{2}$ Center for Emerging Infectious Diseases, University of lowa Research Park,

Coralville, lowa 52241, USA

Full list of author information is available at the end of the article
}

(c) The Author(s). 2019 Open Access This article is distributed under the terms of the Creative Commons Attribution 4.0 International License (http://creativecommons.org/licenses/by/4.0/), which permits unrestricted use, distribution, and reproduction in any medium, provided you give appropriate credit to the original author(s) and the source, provide a link to the Creative Commons license, and indicate if changes were made. The Creative Commons Public Domain Dedication waiver (http://creativecommons.org/publicdomain/zero/1.0/) applies to the data made available in this article, unless otherwise stated. 


\section{Background}

Vector borne zoonotic diseases remain important public health concerns across the globe. No infection occurs in isolation, instead comorbid infection(s) can alter the inflammatory state and subsequently disease outcome [1]. Leishmania spp. are obligate intracellular protozoan parasites which cause a spectrum of diseases, ranging from focal cutaneous lesions to visceralizing disease, endemic within 98 countries across the globe [2]. Visceral leishmaniasis (VL), caused by zoonotic L. infantum, affects nearly 300,000 people each year and leads to more than 30,000 deaths annually. In South America, L. infantum is transmitted both vertically and via phlebotomine sand flies, most commonly Lutzomyia longipalpis [3-5]. In Brazil alone there are more than 3000 new human cases of visceral leishmaniasis each year, with many more likely cases unreported [6]. Disease prevalence in the residents of Natal directly echoes seropositivity of dogs in the area [7], highlighting that disease is often maintained in the canine reservoir and is spread via sand flies to nearby people.

VL is enzootic in Brazil's dog population, the domestic reservoir of L. infantum. Similar to other locations with zoonotic VL [8-10], infected dog ownership remains a significant risk factor predisposing humans to infection [11]. Seroprevalence of canine leishmaniasis (CanL) in Natal has been reported to be between 20-33\% [7, 12]. Because of the important role of dogs as a domestic reservoir for L. infantum in Brazil, current federal public health policy dictates that household dogs are tested for L. infantum via a serological snap test and if positive are required to be donated for euthanasia by the Center of Zoonotic Diseases for each region [13]. Both dogs and humans have a wide range of clinical presentation due to infection with $L$. infantum, ranging from asymptomatic to fatal visceralizing disease. Host and parasite factors that determine clinical outcome are poorly understood. More attention and a better understanding of this neglected disease, particularly the factors that predict clinical outcome, is needed to better control and prevent spread of $L$. infantum.

Within the USA, leishmaniosis is enzootic within the hunting dog population with a qPCR prevalence of $20 \%$, similar to rates of seroprevalence seen in countries with established sand fly-transmitted disease [14, 15]. CanL in the USA is transmitted vertically with no documented vector transmission $[4,16]$. This USA cohort is unique as dogs are exposed to Leishmania parasites in utero then exposed to tick-borne co-infections throughout their lifetime, allowing temporal examination and causal determination of the effects of tick-borne co-infection upon the progression of already present $L$. infantum infection. Tick-borne diseases are prevalent in people and animals across the USA. Prevalence of tick-borne co-infections in this canine research cohort is much greater than that of the USA dog population [17]. For instance, the average seroprevalence of Anaplasma spp. is estimated to be $4.8 \%$ overall in USA dogs [17] while this hunting dog population had a prevalence almost five times higher, near 25\% (Mahachi et al., unpublished data).

The overlapping immune cellular tropisms of tick-borne pathogens and $L$. infantum with alteration to the immune response needed to control of $L$. infantum infection suggests that there is biological plausibility that tick-borne co-infections would alter the immune balance during subclinical VL prompting progression to clinical leishmaniosis.

Co-infection of tick-borne pathogens, common exposures to dogs at high risk of CanL, has been previously reported $[18,19]$. Due to a lack of longitudinal data, previous studies could not assess the causal relationship of comorbid infection to risk of CanL progression [20]. Here we assessed how exposure to tick-borne co-infection altered the clinical outcome of $L$. infantum infection via first a cross-sectional study performed in an endemic area of Brazil. Based on these findings, to solidify the correlation between tick-borne infection and timing of progression to clinical CanL, we obtained temporal data for tick-borne disease exposure and CanL in a longitudinal case-control study. The hypothesis was that dogs exposed to numerous tick-borne co-infections have a higher relative risk of progression to VL.

\section{Methods}

\section{Study design: Brazil}

Two hundred twenty-three dogs were enrolled in a cross-sectional study of household dogs to determine the seroprevalence of tick-borne co-infections with Leishmania spp. in peri-urban areas of Natal, Rio Grande do Norte, Brazil [21, 22]. All dogs were visually assessed by veterinarians and tested serologically for exposure to Anaplasma spp., Ehrlichia spp. and Leishmania spp. All dogs were included in the study with no restrictions. All dogs in the study had similar outdoor exposures and no active intervention for CanL. This is an area, like much of Brazil, where active surveillance is performed to diagnose CanL and the Centro de Controle de Zoonoses (CDZ) euthanizes dogs found to be seropositive for CanL on two tests in accordance with Ministry of Health guidelines.

\section{Study design: USA}

Two hundred eleven dogs, a subset from a block-randomized, double-blinded, placebo-controlled, vaccine trial, were followed longitudinally over a ten-month period (Additional file 1: Figure S1) [23]. Dogs were tested for leishmaniosis via $\mathrm{qPCR}, \mathrm{DPP}^{\circ} \mathrm{CVL}$ assay [24], and a physical exam. Dogs were tested via qPCR and serology for tick-borne disease co-infections. 
Dogs were included in the study if they were older than 6 months of age, not pregnant and not clinically ill with leishmaniasis or any tick-borne disease at the time of study enrollment. All enrollment criteria are the same as our field vaccine trial described in Toepp et al. [25]. Beyond vaccination, which was a covariate analyzed in the present studies, no additional interventions were in place to reduce CanL.

\section{Leishmania status: Brazil}

Exposure to Leishmania spp. was determined through three different serological tests. The Dual Path Platform ${ }^{\circ}$ Canine Visceral Leishmaniasis (DPP ${ }^{\circ} \mathrm{CVL}$ ) assay was used to detect exposure to Leishmania spp. [26]. Two enzyme-linked immunosorbent assays (ELISA) were performed to also identify Leishmania exposure. These two assays targeted recombinant k39 or soluble Leishmania antigens (SLA). Dogs were initially identified as L. infantum-seropositive for univariate analyses if they tested positive on any of these serological assays $\left(\mathrm{DPP}^{\circ} \mathrm{CVL}\right.$ assay, SLA ELISA or k39 ELISA), with additional analyses using the diagnostic step-wise procedure of DPP with confirmative serology to indicate $L$. infantum-positivity [26].

\section{Leishmania status: USA}

Quantitative polymerase chain reaction and the Dual Path Platform ${ }^{\circ}$ Canine Visceral Leishmaniasis (DPP ${ }^{\circ}$ $\mathrm{CVL}$ ) assay were utilized to determine the Leishmania molecular and serological status of dogs in the USA. qPCR and DPP ${ }^{\circledR}$ CVL was performed as in [24].

\section{Physical examination: Brazil}

Veterinarians assessed through visual examination whether dogs had signs of VL. This included onychogryphosis, cachexia, apathy and physical wounds. Blood and serum samples were collected in ethylenediaminetetraacetic acid tubes and stored at $-80{ }^{\circ} \mathrm{C}$. Serum was stored at $-20{ }^{\circ} \mathrm{C}$.

\section{Physical examination: USA}

A veterinarian completed all physical exams. Caretakers provided information on recent travel and hunting activities, use for breeding, and any notable change in the dog's overall health since last visit. If a dog died between visits, a caretaker provided information regarding history of observed clinical signs prior to death. When possible, a full necropsy was performed by a member of the veterinary study team to confirm cause of death with tissue samples submitted for confirmatory Leishmania qPCR. Clinical signs of leishmaniasis included: lymphadenopathy, spleno- and hepatomegaly, epistaxis, alopecia, characteristic macular or papular skin lesions, poor hair coat, cachexia as measured by low body condition score compared to rest of group, conjunctivitis, and onychogryphosis [27, 28].

\section{Outcomes: Brazil}

The objective of the study in Brazil was to determine how exposure to co-infection with tick-borne diseases is associated with exposure to Leishmania spp. In order to assess this relationship Leishmania spp. exposure was the main outcome.

\section{Outcomes: USA}

The objective of the study was to determine how exposure to co-infection with tick-borne diseases affect the progression of leishmaniosis. In order to assess clinical progression of leishmaniosis, dogs were given a clinical score; a combination of VL signs count, DPP ${ }^{\circ} \mathrm{CVL}$, and qPCR for Leishmania. Dogs with three or more clinical signs for $\mathrm{VL}$ who tested positive via $\mathrm{DPP}{ }^{\circ} \mathrm{CVL}$ and/or qPCR for Leishmania were identified as polysymptomatic for CanL. Dogs with two or less clinical signs positive for $\mathrm{DPP}{ }^{\circ} \mathrm{CVL}$ and/or $\mathrm{qPCR}$ were classified as asymptomatic. Dogs negative for both $\mathrm{DPP}^{\circ} \mathrm{CVL}$ and qPCR were classified as negative for CanL. As leishmaniosis is an immunosuppressive disease that is fatal if untreated $[29,30]$ mortality was assessed during the ten-month study. All-cause mortality was defined as mortality for any reason. Licensed veterinarians established leishmaniasis-related deaths by previous Leishmania-specific diagnostic results, history, and clinical signs as well as immediate cause of death (kidney failure etc.).

\section{Measurement of tick co-infections: Brazil}

Serum from dogs were tested for antibody responses to Ehrlichia ewingii and Ehrlichia canis, Anaplasma phagocytophilum and Anaplasma platys, Borrelia burgdorferi and Dirofilaria immitis antigen via the IDEXX SNAP $4 \mathrm{Dx}^{\circ}$ Plus Test. Longitudinal study and confirmation by IDEXX via ELISA was not possible for the Brazil arm of this work, given the need to euthanize any dog that was serologically positive at the (CDZ) in accordance with the Ministry of Health, Brazil.

\section{Measurement of tick co-infections: USA}

Overall the method of establishing tick-borne disease exposure was the same between the two studies, IDEXX SNAP ${ }^{\circ} 4 \mathrm{Dx}^{\circ}$ Plus Test. Blood from dogs was tested via $\mathrm{qPCR}$ for the following tick-borne disease co-infections by IDEXX laboratories: Babesia canis vogeli, Babesia gibsoni, Babesia conradae, Bartonella spp., Rickettsia spp., Hepatozoon americanum, Hepatozoon canis, Ehrlichia canis, Ehrlichia chaffeensis, Ehrlichia ewingii, Anaplasma platys and Anaplasma phagocytophilum. Confirmatory serological testing was performed via ELISA for: speciation for Ehrlichia 
canis, Ehrlichia chaffeensis and Ehrlichia ewingii; speciation for Anaplasma platys and Anaplasma phagocytophilum; speciation for Babesia gibsonii; Borrelia burgdorferi. ELISA results were used for this longitudinal study.

\section{USA data management}

All blood and serum samples were obtained and stored with unique barcode identifiers. All dog names and matching barcode identifier numbers were securely stored on a password-protected network drive in Microsoft Excel spreadsheets and Research Electronic Data Capture, an electronic data capture tool, hosted by University of Iowa, only accessible to a designated research team member, to maintain unbiased physical examination and diagnostic testing.

\section{Statistical analyses: Brazil}

Univariate analyses were performed to determine association between demographic variables and Leishmania spp. seropositivity. Pearson's chi-square test or Fisher's exact test were utilized to assess categorical variables. D’Agostino \& Pearson normality test was performed to determine whether the age variable had a normal distribution. Upon identifying a non-normal distribution the Mann-Whitney test was used to assess age. Logistic regressions were performed to assess the association between tick-borne disease exposure and Leishmania spp. seropositivity controlling for sex, breed, physical appearance and tick serostatus. Older age, male sex, and breeds including Boxers, Italian Spinones, Corsicas, Foxhounds and Beagles have all been shown to have higher risk for Leishmania infection and disease progression [7, 30, 31]. Physical signs of leishmaniosis were included in the regression model to evaluate a correlation between clinical attributes and diagnostic positivity [23, 25]. As the Brazilian Ministry of Health recommends the use of two serological tests to confirm Leishmania spp. seropositivity we included an additional regression analysis utilizing these more stringent recommendations. Statistical analyzes were performed using SAS 9.4 (SAS Institute, Cary, NC, USA), Graph Pad Prism 6 (GraphPad Software Inc., La Jolla, CA, USA), and ArcGIS (Esri, Redlands, CA, USA). Statistical significance was defined as $P$-values at or below 0.05 .

\section{Statistical analyses: USA}

Demographic variables sex and age were compared against clinical leishmaniasis status (Leish+/Leish-) using Pearson's chi-square test of independence. Previous research in our laboratory identified the average age at mortality as six years of age. Age was therefore categorized as dogs six years of age or younger or older than six. Since the observed and expected counts for age $(\leq 6$ years of age/> 6 years of age) were very small, we used Fisher's exact test instead. Odds ratios were computed along with the corresponding 95\% confidence intervals (CI). To further evaluate the association between serological positive tick-borne co-infections and the development of clinical leishmaniosis, a logistic regression was performed with clinical leishmaniosis status as outcome variable. All variables with a $P$-value of less than 0.1 from the univariate analyses were included in the logistic regression as predictor variables. In addition, sex and age were assessed as explanatory variables as both male sex and older age have been reported as potential risk factors related to CanL $[7,32]$. Vaccine status was included as a potential explanatory variable as vaccination could reduce disease progression of CanL [25, 31]. Therefore, sex, age, region, vaccine status and number of serologically positive tick-borne diseases were used as explanatory variables with the outcome of interest as polysymptomatic CanL. Dogs that did not progress to polysymptomatic disease were considered controls.

Dogs that began the study as negative or asymptomatic for either tick-borne or CanL disease were included in this analysis. In the USA, the main route of transmission of CanL is vertical transmission. Due to this route of transmission, dogs are exposed to Leishmania in utero. While both $\mathrm{qPCR}$ and serological tests provide mostly accurate identification of dogs positive for infection/exposure $L$. infantum, there is still the potential for a false negative diagnostic test result. Dogs that began the study as negative and then became positive later may either have had a parasite load/immune response that was below detection during the first time-point or they may have been a true diagnostic false negative. If dogs diagnostically negative at enrollment but living in highly exposed groups were all excluded, the analysis would be biased to only include dogs that were potentially more ill. This would overestimate the true effect of tick exposure upon disease progression.

As this model utilizes longitudinal data, generalized estimating equations (GEE) were used to estimate regression model. An exchangeable correlation matrix structure and logit link function were utilized. Due to the low prevalence of CanL in this population, the rare disease assumption, which states that in cases of rare diseases the odds ratio and relative risk ratio are equivalent, was satisfied and odds ratios were reported as relative risk ratios [33]. Last observation carried forward was used for serological status if dogs died throughout the study and an endpoint leishmaniosis status identified due to mortality. Statistical analyzes were performed using SAS 9.4 (SAS Institute, Cary, NC, USA), Graph Pad Prism 6 (GraphPad Software Inc., La Jolla, CA, USA), and ArcGIS (Esri, Redlands, CA, USA). Statistical significance was defined as $P$-values at or below 0.05 . 


\section{Results}

\section{Brazil study demographics}

Understanding risk factors associated with disease progression and increased transmission are important for any public health control program aimed at reducing transmission from canine reservoirs to people. We performed a cross-sectional study of household dogs around Natal, Brazil, to determine the frequency and association of exposure to tick-borne diseases and the outcome of seropositive L. infantum infection. Blood/ serum samples were collected from 223 dogs within a lower socioeconomic area of Natal, Brazil. Age and breed information was collected from dog owners. The average age was 2.5 years-old with a range from 3 months to 13 years (Table 1 ). Only a quarter of dogs were identified by veterinarians as showing visible signs of VL. The most common sign was onychogryphosis (17\%), other signs were seen in less than $10 \%$ of dogs. Tick-borne disease exposure was high. Ehrlichia spp. was the most common tick-borne disease exposure. Leishmania spp. seropositivity was high with $50 \%$ of dogs testing seropositive on one or more tests (Fig. 1a).

To better understand the association between tick-borne disease exposure and Leishmania infection in Brazilian dogs, a univariate analysis assessing each demographic variable in relationship to the outcome of Leishmania spp. seropositivity was performed (Table 2). Our previous research has shown with a cohort of 130 dogs from the USA that when using ELISA as a gold standard, the sensitivity and specificity of the $\mathrm{DPP}{ }^{\circ} \mathrm{CVL}$ is $73 \%$ and $80 \%$, respectively [24]. Furthermore, several additional studies corroborate these findings and have shown that these type of diagnostic tests correlate [34-37]. Therefore, DPP ${ }^{\circ} \mathrm{CVL}$ assay results were used as a proxy for Leishmania spp. seropositivity in this analysis. The risk of positive Leishmania spp. serostatus based on tick-borne disease exposure was statistically significant. The risk of Leishmania spp. seropositivity was significantly greater among dogs with any tick exposure $v s$ dogs with no tick exposures (RR: 1.55, $P=0.0135)$ (Fig. 1b, c).

\section{Tick-borne disease exposure is significantly associated with Leishmania spp. exposure in a dose-dependent} manner

We performed a logistic regression assessing the outcome of seropositivity for Leishmania spp. using the number of tick-borne disease exposures, age, sex, breed, appearance of onychogryphosis, cachexia, physical wounds, or apathy as covariates (Table 3). Only dogs with complete data for all explanatory variables were included in the analysis (included 208/223 dogs). Dogs exposed to Ehrlichia spp. and Anaplasma spp. were $2.69 \times$ more likely to also be seropositive for Leishmania spp. (Adjusted RR: 1.68, 95\% CI: 1.09-2.61, $P=0.019)$ than dogs not exposed to tick-borne
Table 1 Demographics of Brazil cross-sectional study cohort

\begin{tabular}{|c|c|}
\hline $\begin{array}{l}\text { Variable } \\
\text { nat }\end{array}$ & Value, $n(\%)$ \\
\hline Sex, $n$ (\% male) & $115(51.57)$ \\
\hline Age, mean \pm SD (range) (years) & $2.48 \pm 2.16(0.25-13.00)$ \\
\hline Breed, $n$ (\% mixed breed) & $188(90.38)$ \\
\hline \multicolumn{2}{|l|}{ Appearance } \\
\hline Onychogryphosis & $40(17.94)$ \\
\hline Cachexia & $19(8.52)$ \\
\hline Wounds/skin lesions & $18(8.07)$ \\
\hline Apathy & $4(1.79)$ \\
\hline None & $171(76.68)$ \\
\hline \multicolumn{2}{|l|}{ Tick serology } \\
\hline Ehrlichia spp. & $91(40.81)$ \\
\hline Anaplasma spp. & $1(0.45)$ \\
\hline Anaplasma spp. + Ehrlichia spp. & $73(32.74)$ \\
\hline None & $58(26.01)$ \\
\hline \multicolumn{2}{|l|}{ Leishmania spp. serology } \\
\hline \multicolumn{2}{|l|}{ Seropositive: three tests } \\
\hline DPP'CVL, SLA, rk39 & $12(5.38)$ \\
\hline \multicolumn{2}{|l|}{ Seropositive: two tests } \\
\hline DPP ${ }^{\circledR} \mathrm{CVL}$ and SLA & $17(7.62)$ \\
\hline $\mathrm{DPP}^{\circledR} \mathrm{CVL}$ and rk39 & $19(8.52)$ \\
\hline \multicolumn{2}{|l|}{ Seropositive: one test } \\
\hline $\mathrm{DPP}{ }^{\oplus} \mathrm{CVL}$ & $108(48.43)$ \\
\hline SLA & $19(8.52)$ \\
\hline rk39 & $22(9.87)$ \\
\hline Seronegative (all tests) & $111(49.78)$ \\
\hline
\end{tabular}

Abbreviation: $S D$ standard deviation

disease. Dogs seropositive for Ehrlichia spp. alone or Anaplasma spp. alone were also statistically significantly more likely to be seropositive for Leishmania spp. than non-tick-borne disease seropositive dogs (Adjusted RR: 1.60, 95\% CI: 1.04-2.45, $P=0.032$ ). Using the Brazilian Ministry of Health guidelines, i.e. reactivity on two serological tests as seropositive for Leishmania spp., dogs seropositive for one tick-borne disease were at a significantly higher risk of being Leishmania spp.-seropositive (Adjusted RR: 4.86, 95\% CI: $1.16-20.3, P=0.030$ ) (Table 3 ). This significant association indicates that dogs from northeastern Brazil exposed any tick-borne disease are more likely to be seropositive for Leishmania.

\section{Longitudinal study of tick-borne co-infection in $L$. infantum-infected dogs}

Based on these findings in Brazilian dogs, indicating that there was substantial exposure to tick-borne diseases, and that this exposure increased the risk of seropositive $\mathrm{VL}$, we were interested in learning whether this was a causal relationship. Specifically, we wanted to test in a 


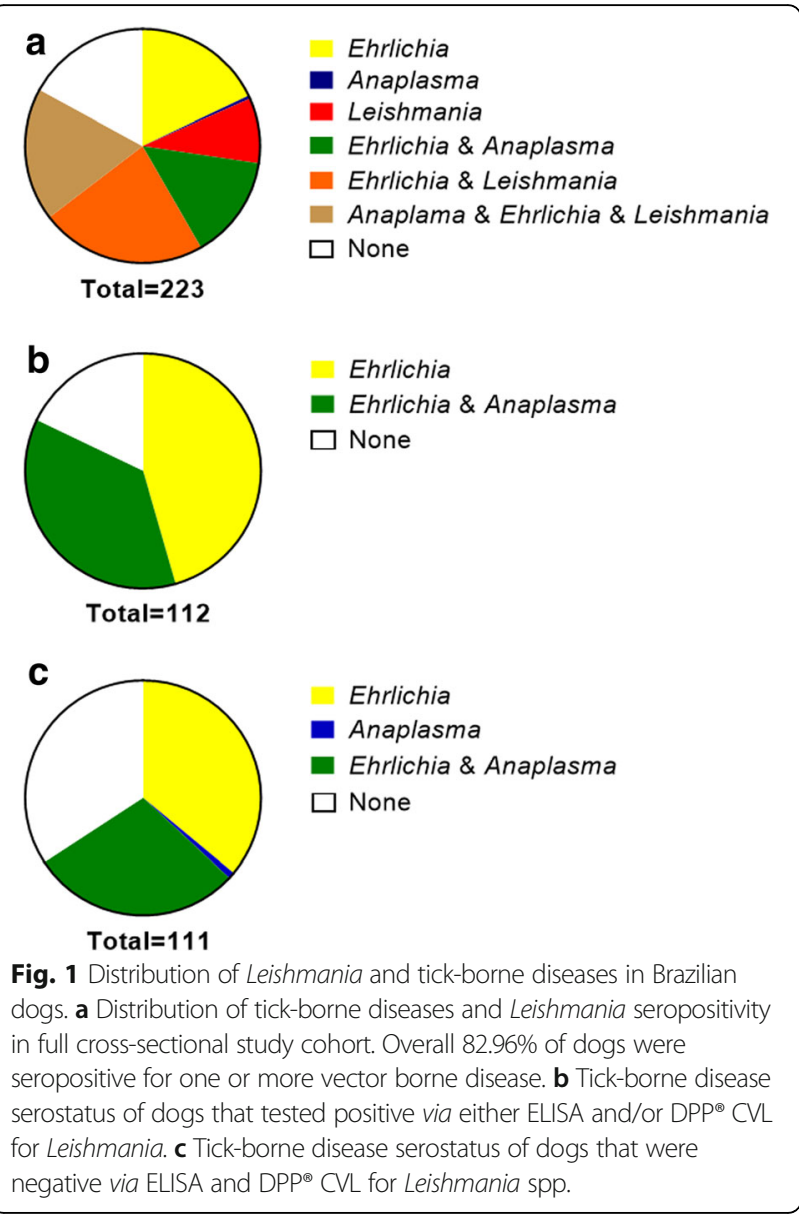

setting where dogs were already infected with $L$. infantum how exposure to tick-borne diseases altered the risk of progression to CanL. As we have access to a large cohort of USA dogs infected in utero with $L$. infantum $[4,38]$ and highly exposed to tick-borne diseases, we used this group of dogs to follow longitudinal exposure to tick-borne disease and progression with VL. Two hundred eleven dogs were enrolled in a longitudinal nested case-control study stemming from a larger vaccine trial [25]. Dogs positive for a tick-borne infection via the 4DXSnap Plus test were on average 4.53 years-old with a standard deviation of 2.05 years, while dogs that were identified as tick disease-negative were slightly younger (3.80 years, SD 2.41 years, Table 4 ). No significant differences between age groups based on tick serology or age group based on sex were identified (Additional file 1: Figure S2). A univariate analysis of dogs that progressed to symptomatic disease, diagnostically positive with two or more clinical signs, $v s$ those that did not, was performed. This analysis showed that dogs with historical exposure to two or more tick-borne disease co-infections via ELISA were 3.39 times more likely to have clinical signs for leishmaniosis (95\% CI: 1.174-9.413, $P=0.0381$ ). It was also seen that dogs older than six years
Table 2 Univariate analysis of study cohort variables based on Leishmania serostatus. Pearson's chi-square test and ANOVA were used to analyze categorical variables where appropriate

\begin{tabular}{|c|c|c|c|}
\hline Variable & $\begin{array}{l}\text { Leishmaniat } \\
(n=108)\end{array}$ & $\begin{array}{l}\text { Leishmania- } \\
(n=115)\end{array}$ & $\overline{P \text {-value }}$ \\
\hline$\overline{\text { Sex }, n(\% \text { male })}$ & $54(24.2)$ & $61(27.6)$ & 0.6886 \\
\hline $\begin{array}{l}\text { Age, mean } \pm \text { SD (range) } \\
\text { (years) }\end{array}$ & $\begin{array}{l}2.45 \pm 1.81 \\
(0.42-8.00)\end{array}$ & $\begin{array}{l}2.50 \pm 2.45 \\
(0.25-13.0)\end{array}$ & \\
\hline Breed, $n$ (\% mixed breed) & $11(5.29)$ & $9(4.33)$ & 0.6392 \\
\hline \multicolumn{4}{|l|}{ Appearance, $n(\%)$} \\
\hline Onychogryphosis & $21(19.4)$ & $19(16.5)$ & 0.3691 \\
\hline Cachexia & $8(7.41)$ & $11(9.57)$ & \\
\hline Physical wounds & $9(8.33)$ & $9(7.83)$ & \\
\hline Apathy & $0(0)$ & $4(3.48)$ & \\
\hline None & $81(75)$ & $90(78.3)$ & \\
\hline \multicolumn{4}{|l|}{ Tick disease exposure, $n$ (\%) } \\
\hline Ehrlichia alone & $88(81.5)$ & $76(66.1)$ & 0.0290 \\
\hline Anaplasma alone & $39(36.1)$ & $35(30.4)$ & \\
\hline Anaplasma + Ehrlichia & $39(36.1)$ & $34(29.6)$ & \\
\hline None & $20(18.5)$ & $38(33.0)$ & \\
\hline
\end{tabular}

Notes: Age differences assessed via Mann-Whitney test. Leishmania+ are dogs that tested positive via the DPP ${ }^{\oplus} \mathrm{CVL}$ assay. Leishmania- are dogs that tested negative via the $\mathrm{DPP}^{\otimes} \mathrm{CVL}$ assay

Abbreviation: $S D$ standard deviation

of age were 3.62 times more likely to have clinical signs (95\% CI: 1.427-8.715, $P=0.0055$ ) (Table 5).

Tick-borne disease exposure was significantly associated with clinical leishmaniosis

To establish the effect of tick-borne disease on the outcome of CanL controlling for all other important variables, a logistic regression analysis was performed on the longitudinal data. Only dogs that began the study as negative or asymptomatic for CanL were included in this analysis to address progression; 203 dogs were included. Ehrlichiosis, Lyme disease, anaplasmosis and CanL have some overlapping clinical signs [38-40]. Due to this, only dogs identified as polysymptomatic for leishmaniosis, three or more clinical signs specific to leishmaniosis, were considered as having progressed to CanL.

Adjusting for other variables and using a more stringent definition for clinical CanL, dogs with three or more tick-borne diseases have 11 times greater odds of being polysymptomatic for leishmaniosis than dogs with no exposure to tick-borne diseases (Adjusted Relative Risk, ARR: $11.65,95 \%$ CI: $1.22-110.99, P=0.033$ ) (Table 6). We found that this association was dose dependent; exposure to more tick-borne diseases increased the risk of progression to clinical CanL within the time of the study, an important feature in indicating a causal relationship. The odds of being polysymptomatic for CanL were 7.69 times greater for dogs exposed to three tick-borne diseases 
Table 3 Dogs seropositive for a tick-borne disease are more likely to be seropositive for Leishmania. Parameter estimates were determined using logistic regression

\begin{tabular}{|c|c|c|c|}
\hline Variable & ARR & $95 \% \mathrm{Cl}$ & $P$-value \\
\hline \multicolumn{4}{|c|}{ (i) Leish serology 1 test } \\
\hline \multicolumn{4}{|l|}{ Sex } \\
\hline Male vs female & 0.97 & $0.74-1.28$ & 0.85 \\
\hline Age & 0.97 & $0.91-1.04$ & 0.38 \\
\hline \multicolumn{4}{|l|}{ Appearance } \\
\hline Onychogryphosis & 1.22 & $0.84-1.76$ & 0.29 \\
\hline Cachexia & 0.99 & $0.54-1.80$ & 0.97 \\
\hline Physical wounds & 1.11 & $0.71-1.74$ & 0.63 \\
\hline Apathy & 0.35 & $0.60-2.05$ & 0.24 \\
\hline \multicolumn{4}{|l|}{ Tick disease serostatus } \\
\hline positive for 1 vs 0 & 1.60 & $1.04-2.45$ & 0.032 \\
\hline positive for 2 vs 0 & 1.68 & $1.09-2.61$ & 0.019 \\
\hline positive for 1 vs 2 & 0.95 & $0.70-1.29$ & 0.73 \\
\hline \multicolumn{4}{|l|}{ Breed } \\
\hline Mixed vs Purebred & 0.99 & $0.63-1.54$ & 0.95 \\
\hline \multicolumn{4}{|c|}{ (ii) Leish serology 2 tests } \\
\hline \multicolumn{4}{|l|}{ Sex } \\
\hline Male vs female & 0.56 & $0.26-1.20$ & 0.14 \\
\hline \multicolumn{4}{|l|}{ Tick disease serostatus } \\
\hline positive for 1 vs 0 & 4.86 & $1.16-20.3$ & 0.030 \\
\hline positive for 2 vs 0 & 2.75 & $0.60-12.7$ & 0.19 \\
\hline positive for 1 vs 2 & 1.76 & $0.77-4.05$ & 0.18 \\
\hline
\end{tabular}

a Bold indicates statistically significant variables

Notes: Leishmaniosis 1 test: Leishmania-positive are dogs that tested positive via DPP ${ }^{\circledR} \mathrm{CVL}$ assay or ELISA. Leishmania-negative are dogs that tested negative via the DPP ${ }^{\oplus} \mathrm{CVL}$ assay and ELISA. Predictor variables for this model included age, sex, appearance, tick disease status, and breed. Leishmaniosis 2 tests: Leishmania-positive are dogs that tested positive via the DPP ${ }^{\oplus} \mathrm{CVL}$ assay and ELISA. Leishmania-negative are dogs that tested negative via the DPP ${ }^{\circledR} \mathrm{CVL}$ assay and ELISA. Predictor variables for this model included sex and tick disease serostatus

Abbreviations: $\mathrm{Cl}$ confidence interval, $A R R$ adjusted relative risk

Table 4 Demographics of longitudinal study cohort. Combined IDEXX SNAP ${ }^{\oplus} 4 D X^{\circledast}$ test results for antibody against Ehrlichia ewingii and Ehrlichia canis, Anaplasma phagocytophilum and Anaplasma platys, Borrelia burgdorferi, and antigen to Dirofilaria immitis

\begin{tabular}{lll}
\hline Variable & $\begin{array}{l}\text { IDEXX SNAP } \\
\text { (exposed) }\end{array}$ & $\begin{array}{l}\text { IDEX } \\
\text { (unexposed) }\end{array}$ \\
\hline No. & 62 & 149 \\
Age, \% $\leq$ 6 years-old & 79.03 & 88.51 \\
Sex, \% male & 50.00 & 51.68 \\
\hline
\end{tabular}

Table 5 Univariate analysis of factors leading to CanL from longitudinal study

\begin{tabular}{lllll}
\hline Variable & $\begin{array}{l}\text { Leish+ } \\
(n=16)\end{array}$ & $\begin{array}{l}\text { Leish- } \\
(n=195)\end{array}$ & RR & P-value \\
\hline Age, \% $\leq$ years old & 62.50 & 87.69 & 3.62 & 0.0055 \\
Sex, \% male & 43.75 & 51.79 & 0.7418 & 0.5360 \\
Tick serology-historical exposure & & & 3.388 & 0.0381 \\
$\quad$ 2 or more & 41.67 & 15.87 & & \\
$\quad$ less than 2 & 58.33 & 84.13 & & \\
Treatment group & 50.00 & 51.31 & 0.9519 & $>0.9999$ \\
Placebo vs vaccine & 50.00 & 48.69 & & \\
\hline
\end{tabular}

Abbreviations: CanL, canine leishmaniosis. Leish+, showing 2 or more clinical signs of canine leishmaniasis; Leish-, showing less than two clinical signs of canine leishmaniasis or negative for $L$. infantum via $\mathrm{APCR}$ or DPP ${ }^{\circledR} \mathrm{CVL}$ assay; $\mathrm{RR}$, unadjusted relative risk

(or more) compared to those exposed to the group of dogs with only one tick-borne disease (ARR: 7.69, 95\% CI: 1.39-43.48, $P=0.0200)$. Dogs exposed to three tick-borne diseases also saw a significant increase in the odds of being polysymptomatic with CanL compared to dogs with two tick-borne diseases (ARR: 8.33, 95\% CI: 1.01-66.67, P $=0.0490)$.

\section{Dogs with tick-borne diseases and Leishmania were significantly more likely to die}

Antidotal evidence indicated that dogs with tick-borne disease diagnosis and a history of being diagnostically positive for CanL seemed to correlate strongly with a high mortality rate. Based on the proposed relationship

Table 6 More exposure to multiple tick-borne diseases leads to worse CanL. Multiple logistic regression with outcome defined as polysymptomatic leishmaniosis; dogs diagnostically positive for Leishmania infantum with three or more specific signs for leishmaniosis

\begin{tabular}{llll}
\hline Variable & $\begin{array}{l}\text { Adjusted } \\
\text { relative risk }\end{array}$ & $95 \% \mathrm{Cl}$ & $P$-value \\
\hline Age & & & \\
$\leq 6$ years-old vs older & 8.50 & $2.22-32.51$ & 0.0018 \\
$\begin{array}{l}\text { Region } \\
\text { Mid-west vs other }\end{array}$ & 11.78 & $1.79-77.57$ & 0.010 \\
Serology (no. of tick-borne dz) & & \\
1 vs 0 & 1.49 & $0.29-7.79$ & 0.630 \\
2 vs 0 & 1.44 & $0.25-8.40$ & 0.690 \\
3 vs 0 & 11.65 & $1.22-110.99$ & 0.033 \\
3 vs 1 & 7.69 & $1.39-43.48$ & 0.020 \\
3 vs 2 & 8.33 & $1.01-66.67$ & 0.049 \\
2 vs 1 & 1.04 & $0.27-3.96$ & 0.950 \\
\hline
\end{tabular}

Notes: Explanatory variables controlled for in the model were age, region, tick serological status (positive vs negative), sex and vaccine status. Only significant variables are shown

Abbreviations: CanL, canine leishmaniosis; $\mathrm{Cl}$, confidence interval; $\mathrm{dz}$, disease 
between progression of clinical CanL after exposure to tick-borne diseases, we were interested to see how dogs diagnostically positive for Leishmania spp. and positive via ELISA for a tick-borne disease compared to those without these exposures in terms of all-cause mortality. Dogs diagnostically positive for Leishmania spp. and positive via ELISA for a tick-borne disease were 4.85 times more likely to die than those that negative for both (RR: 4.85, 95\% CI: $1.65-14.24, P=0.0051)$. When controlling for age, this relationship remained significant in dogs that were six years of age or younger (4.74 times more likely to die from all causes within one year (RR: 4.74, 95\% CI: $1.32-16.88, P=0.027)$ ). This significant association between tick-borne disease exposure and CanL outcome or mortality can also be seen graphically (Fig. 2).

\section{Ehrlichia spp. and Anaplasma spp. cause most common tick-borne disease in dogs with clinical CanL}

The most common tick-borne infection was Babesia spp. with an overall average seroprevalence of $31.65 \%$. When evaluating dogs with clinical CanL (two or more clinical signs), Ehrlichia spp. and Anaplasma spp. were the most common tick-borne disease agent exposures among dogs with clinical CanL, of note also the two most common tick-borne disease agent exposures in Brazil as well. Leishmania spp. while only found in $17-20 \%$ of the study cohort depending on the time of the year was the most common exposure/infection found within dogs that died with more than $50 \%$ of the dogs that died being diagnostically positive for Leishmania spp. The second most common was Babesia spp. present in $27 \%$ of dogs that died (Table 7).

\section{Other tick-borne infections}

Bartonella spp. and Rickettsia exposure was found in the longitudinal study cohort as well. Three dogs were positive for Bartonella spp. infection via PCR at enrollment. All dogs were male, under the age of six, two of the dogs were from eastern USA, and one was from the Midwest. One dog of these dogs was also exposed to Anaplasma spp. and Ehrlichia spp.; another to Anaplasma spp., Borrelia burgdorferi, and Babesia spp., and one dog had been exposed to Anaplasma spp. and Babesia spp. The two dogs that tested positive for Rickettsia spp. via PCR tested positive in August were male and under the age of six from the Eastern USA. One dog was exposed to Anaplasma spp., Borrelia burgdorferi and Leishmania spp. The other dog was exposed to both Borrelia burgdorferi and Leishmania spp.

\section{Discussion}

Co-infections can increase disease severity as is seen in both canine and human patients in Brazil with co-infections in addition to Leishmania infection [41, 42]. In Brazil, people infected with both Leishmania and human immunodeficiency virus (HIV) had increased mortality [43, 44], much like dogs in this study with tick coinfection and CanL. A similar trend was seen in human patients with Lyme disease and babesiosis co-infection [45-47]. Increased disease severity and pathogenicity was found in patients co-infected with Leishmania and intestinal helminths [48]. Malnutrition is also a risk factor of leishmaniasis stressing the importance of understanding these diseases within areas of lower socioeconomic status where malnutrition is common [49]. In canine patients, higher morbidity and mortality between vector borne diseases was observed in multiple case reports
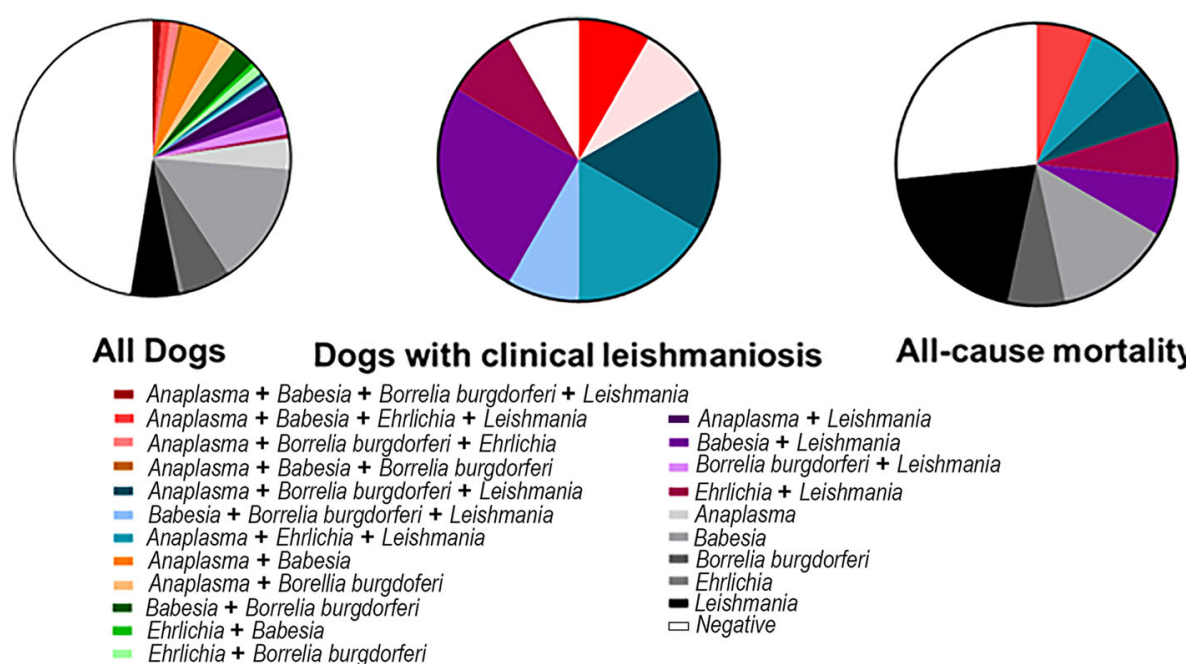

Fig. 2 Distribution of tick-borne diseases based on CanL clinical status or mortality. Tick-borne disease exposure determined as positive or negative via ELISA. Leishmania spp. determined as diagnostically positive via qPCR and/or DPP® CVL. a Dogs with clinical leishmaniosis determined as dogs with three or more clinical signs of leishmaniosis and diagnostically positive for Leishmania via qPCR and/or DPP ${ }^{\circ}$ CVL. b All-cause mortality determined as death for any reason 
Table 7 Distribution of co-infections among dogs with clinical leishmaniosis and that died

\begin{tabular}{lll}
\hline Tick-borne exposure & \% also CanL & $\begin{array}{l}\text { \% all-cause } \\
\text { mortality }\end{array}$ \\
\hline Anaplasma spp. & 41.67 & 20.00 \\
Babesia spp. & 50.00 & 26.67 \\
Borrelia burgdorferi & 33.33 & 13.33 \\
Ehrlichia spp. & 41.67 & 20.00 \\
Leishmania spp. & 100 & 53.33
\end{tabular}

Notes: Leishmania infantum positive status was determined as any dog that was diagnostically positive via $\mathrm{APCR}$ or is measured by either DPP ${ }^{\circledR} \mathrm{CVL}$ assay or PCR-positive. Clinical leishmaniosis was determined as dogs with 2 or more clinical signs for leishmaniosis and a diagnostic positive test results on $\mathrm{qPCR}$ and/or DPP ${ }^{\oplus} \mathrm{CVL}$

and experimental infection studies including co-infection with Anaplasma spp. and Ehrlichia spp. as well as Babesia spp. and Leishmania spp. [50, 51]. Recent studies in Europe have identified significant associations between CanL and other vector borne diseases $[18,20,52]$. These studies have been limited by greater complication of establishing temporality when there is vector borne transmission [7]. Most, if not all, dogs in the longitudinal USA study were vertically exposed to leishmaniosis, with limited exposure after gestation. This greatly improves the ability to know what disease came first $[3,4,38]$. Other routes of transmission have been suggested including sexual transmission or a possible role of ticks in transmission. As ticks are not biological vectors for L. infantum, as compared to sand flies, their only role could be in mechanical transmission and multiple incomplete feedings by a tick. There is no evidence that supports tick-borne transmission of $L$. infantum.

The aim of this study was to determine whether there is a significant, causal relationship between tick-borne disease exposure and the development of VL in dogs, the domestic reservoir for infection in Brazil. We found that having multiple tick-borne co-infections, which was common in Brazilian dogs, significantly increased the risk of CanL progression. This relationship was dose-dependent, i.e. the more tick-borne co-infections a dog had, the higher the risk of CanL progression and mortality. Ninety-two percent of dogs with clinical leishmaniosis were positive for a tick-borne disease and $58 \%$ of dogs with clinical leishmaniosis were positive for two or more tick-borne diseases. Thirty-three percent of dogs that died had two or more co-infections. These results provide epidemiological evidence that co-infection with a tick-borne disease may offset the delicate balance within the immune system during Leishmania infantum infection. Based on these findings, in many endemic/enzootic areas, times of great tick-borne disease prevalence could potentially drive the occurrence of more L. infantum parasitemic dogs and greater L. infantum transmission to people. Conversely, based on this causal relationship between tick-borne disease exposure and clinical CanL, controlling tick-borne disease may also control CanL.

Ehrlichia spp. and Anaplasma spp. were the most common tick-borne disease exposures within dogs that progressed to clinical disease found in over $40 \%$ of the dogs. This could be because Ehrlichia spp. can infect macrophages and can lead to remarkable proinflammatory then regulatory immunity disrupting the Th1 response needed for control of CanL [53-56]. Once inside phagocytes Ehrlichia spp. can downregulate IL-12, important for activation of CD4 T cells to release IFN- $\gamma$ important in upregulating macrophage killing to remove Ehrlichia spp. and Leishmania infantum $[57,58]$. Ehrlichia spp. can reduce autophagy allowing bacteria and Leishmania spp. to continue growing and replicating within the cell [59]. These co-infections have been shown to alter host immunity in ways that would allow $L$. infantum infection to thrive within phagocytes leading to clinical disease.

This study highlights the need to address reducing tick-borne disease exposure and co-infection rates in order to reduce clinical progression and its important correlate, transmission of Leishmania infantum. Xenodiagnosis studies addressing CanL transmission to the sand flies suggested that clinically apparent dogs were more likely to transmit $L$. infantum to the sand flies [60-62]. If tick-borne disease prevention and treatments can be utilized to reduce leishmaniosis disease progression, there may also be a correlation in reducing of Leishmania infantum transmission. These aspects need to be addressed for control and elimination of the disease in both human and animal populations.

This study was limited in the length of time of follow-up. Additional studies following dogs for a longer would allow evaluation of long-term effects of tick exposure over multiple seasons. Loss to follow up was also a limitation of the study as dogs were lost to follow-up for many reasons including drafting and retirement, which limited the ability to identify all dogs that became clinically ill. The loss to follow up likely lead to an underestimation of the true association, as dogs that were drafted were likely underperforming potentially due to illness.

\section{Conclusions}

Significant associations between exposure to tick-borne infectious agents and progression of CanL were found in this study. These findings will require additional immunological studies to identify the specific immune mechanisms of how tick-borne diseases affect progression of CanL. Future studies could lead to targets for potential treatments and immunotherapies based on the loss of immunoregulation that may be associated with tick-borne coinfection during CanL. Based on the association of tick-borne disease with CanL progression, tick prevention and risk management could be realistic tools to reduce the seroprevalence of CanL within hunting dogs. 


\section{Additional files}

Additional file 1: Figure S1. US hunting dog longitudinal study timeline. Dogs were sampled three times designated by up and down arrows, over a tick season. Peak tick season for all kennel locations is designated by black. The bridge to tick season, dependent on seasonal variation and geographic location, shown in grey. Figure S2. Age and sex distribution of dogs based on tick-borne disease exposure at enrollment. Tick exposure based on SNAP ${ }^{\circledR}$ 4Dx ${ }^{\circledR}$ Plus Test. a Age distribution. b Sex distribution. Abbreviations: CanL: canine leishmaniosis; qPCR: quantitative polymerase chain reaction; RR: risk ratio; ARR: adjusted risk ratio; OR: odds ratio; Cl: confidence interval. (ZIP 212 kb)

\section{Acknowledgements}

The authors would like to thank the Centro de Controle de Zoonoses of Rio Grande do Norte for their assistance throughout this project and IDEXX Laboratories Inc. for providing the IDEXX SNAP ${ }^{\otimes} 4 D X^{\circledast}$ Plus Test. We would also like to thank the owners of the hunting dogs for their participation in this study and multiple veterinarians for helping us obtain the samples upon which this work is based; Rachel Westerlund, Jane Jeffries, Caitlin Cotter, Radhika Gharpure, George Seier, Marvin Beeman, and many others for providing us support during these studies.

\section{Funding}

This work was funded by grant D16CA-517 from the Morris Animal Foundation with support from the Masters of Foxhounds Association Foundation and by R01 TW 010500 through the Fogarty International Center of the US National Institutes of Health. Neither of the funding agencies had a role in the study design or manuscript preparation.

\section{Availability of data and materials}

The data supporting the conclusions of this article are included within the article and its additional file. The full dataset is available at https://www.researchgate.net/ publication/330260113_Tick_borne_disease_associated_with_progression_of_ Can_L_Petersen_lab_IDEXX_data

\section{Authors' contributions}

AJT performed conceptualization/methodology, investigation, formal analysis, manuscript writing and figure development. KM performed formal analysis, GW TGP, CB, MA, HF and MP performed investigation, BA performed investigation and formal analysis, ALL, GRGM, JFVC, KW, JS, PT and ML performed investigation, ZP performed figure development, JB performed investigation and assisted with methodology, RC assisted with conceptualization/ methodology. JJO assisted in conceptualization/ methodology and analysis development. GDB and MVO assisted in methodology and analysis development. SBMJ provided conceptualization/ methodology, investigation and editing of the manuscript. CAP provided conceptualization/ methodology, investigation, writing and editing of the manuscript. All authors read and approved the final manuscript.

\section{Ethics approval}

All dogs were enrolled with informed consent from their caretakers and all protocols followed were approved by the University of lowa Institution Animal Care and Use Committee (IACUC) an AAALAC accredited institution and the Universidade de Federal de Rio Grande do Norte Committee for Ethical Use of Animals (CEUA) following the requirements for the US National Institutes of Health Office of Laboratory Animal Welfare Assurances which operates under the 2015 reprint of the Public Health service Policy on Humane care and Use of Laboratory Animals.

\section{Consent for publication}

Not applicable.

\section{Competing interests}

The authors declare that they have no competing interests.

\section{Publisher's Note}

Springer Nature remains neutral with regard to jurisdictional claims in published maps and institutional affiliations.

\section{Author details}

'Department of Epidemiology, College of Public Health, University of lowa, lowa City, lowa 52242, USA. ${ }^{2}$ Center for Emerging Infectious Diseases, University of lowa Research Park, Coralville, lowa 52241, USA. ${ }^{3}$ Institute of Tropical Medicine of Rio Grande do Norte, Federal University of Rio Grande do Norte, Natal, RN 59078-970, Brazil. ${ }^{4}$ Department of Biostatistics, College of Public Health, University of lowa, lowa City, lowa 52242, USA. ${ }^{5}$ Immunology Program, Carver College of Medicine, University of lowa, lowa City, lowa 52242, USA. ${ }^{6}$ IDEXX Laboratories Inc., One IDEXX Drive, Westbrook, Maine 04092, USA. ${ }^{7}$ Department of Geographical and Sustainability Sciences, College of Liberal Arts \& Sciences, University of lowa, lowa City, lowa 52242, USA.

Received: 16 July 2018 Accepted: 13 January 2019

Published online: 23 January 2019

\section{References}

1. Vorou RM, Papavassiliou VG, Tsiodras S. Emerging zoonoses and vectorborne infections affecting humans in Europe. Epidemiol Infect. 2007;135: 1231-47.

2. Alvar J, Velez ID, Bern C, Herrero M, Desjeux P, Cano J, et al. Leishmaniasis worldwide and global estimates of its incidence. PLoS One. 2012;7:e35671.

3. Grinnage-Pulley T, Scott B, Petersen CA. A mother's gift: congenital transmission of Trypanosoma and Leishmania species. PLoS Pathog. 2016;12: e1005302.

4. Boggiatto PM, Gibson-Corley KN, Metz K, Gallup JM, Hostetter JM, Mullin $\mathrm{K}$, et al. Transplacental transmission of Leishmania infantum as a means for continued disease incidence in North America. PLoS Negl Trop Dis. 2011;5:e1019.

5. Ximenes MF, Castellon EG, de Souza MF, Freitas RA, Pearson RD, Wilson $M E$, et al. Distribution of phlebotomine sand flies (Diptera: Psychodidae) in the state of Rio Grande do Norte, Brazil. J Med Entomol. 2000;37: 162-9.

6. de Melo EC, Fortaleza CM. Challenges in the therapy of visceral leishmaniasis in Brazil: a public health perspective. J Trop Med. 2013; 2013:319234.

7. Lima ID, Lima ALM, Mendes-Aguiar CO, Coutinho JFV, Wilson ME, Pearson RD, et al. Changing demographics of visceral leishmaniasis in northeast Brazil: lessons for the future. PLoS Negl Trop Dis. 2018;12: e0006164.

8. Aoun O, Mary C, Roqueplo C, Marie JL, Terrier O, Levieuge A, et al. Canine leishmaniasis in south-east of France: screening of Leishmania infantum antibodies (western blotting, ELISA) and parasitaemia levels by PCR quantification. Vet Parasitol. 2009;166:27-31.

9. Biglino A, Bolla C, Concialdi E, Trisciuoglio A, Romano A, Ferroglio E. Asymptomatic Leishmania infantum infection in an area of northwestern Italy (Piedmont region) where such infections are traditionally nonendemic. J Clin Microbiol. 2010;48:131-6.

10. Hajjaran H, Mohebali M, Mamishi S, Vasigheh F, Oshaghi MA, Naddaf SR, et al. Molecular identification and polymorphism determination of cutaneous and visceral leishmaniasis agents isolated from human and animal hosts in Iran. BioMed Res Int. 2013;2013:789326.

11. Lima ALM, de Lima ID, Coutinho JFV, de Sousa U, Rodrigues MAG, Wilson $M E$, et al. Changing epidemiology of visceral leishmaniasis in northeastern Brazil: a 25-year follow-up of an urban outbreak. Trans R Soc Trop Med Hyg. 2017;111:440-7.

12. Queiroz PV, Monteiro GR, Macedo VP, Rocha MA, Batista LM, Queiroz JW, et al. Canine visceral leishmaniasis in urban and rural areas of Northeast Brazil. Res Vet Sci. 2009:86:267-73.

13. Ashford DA, David JR, Freire M, David R, Sherlock I, Eulalio MC, et al. Studies on control of visceral leishmaniasis: impact of dog control on canine and human visceral leishmaniasis in Jacobina, Bahia, Brazil. Am J Trop Med Hyg. 1998:59:53-7.

14. Toepp AJ, Schaut RG, Scott BD, Mathur D, Berens AJ, Petersen CA. Leishmania incidence and prevalence in U.S. hunting hounds maintained via vertical transmission. Vet Parasitol Reg Stud Rep. 2017; 10:75-81

15. Coura-Vital W, Marques MJ, Veloso VM, Roatt BM, Aguiar-Soares RD, Reis LE, et al. Prevalence and factors associated with Leishmania infantum infection of dogs from an urban area of Brazil as identified by molecular methods. PLoS Negl Trop Dis. 2011;5:e1291. 
16. Schaut RG, Robles-Murguia M, Juelsgaard R, Esch KJ, Bartholomay LC, Ramalho-Ortigao M, et al. Vectorborne transmission of Leishmania infantum from Hounds, United States. Emerg Infect Dis. 2015;21:2209-12.

17. McMahan CS, Wang D, Beall MJ, Bowman DD, Little SE, Pithua PO, et al. Factors associated with Anaplasma spp. seroprevalence among dogs in the United States. Parasit Vectors. 2016:9:169.

18. Baxarias M, Alvarez-Fernandez A, Martinez-Orellana P, Montserrat-Sangra $S$, Ordeix $L$, Rojas $A$, et al. Does co-infection with vector-borne pathogens play a role in clinical canine leishmaniosis? Parasit Vectors. 2018;11:135.

19. Mekuzas Y, Gradoni L, Oliva G, Foglia Manzillo V, Baneth G. Ehrlichia canis and Leishmania infantum co-infection: a 3-year longitudinal study in naturally exposed dogs. Clin Microbiol Infect. 2009;15(Suppl. 2):30-1.

20. Attipa C, Solano-Gallego L, Papasouliotis K, Soutter F, Morris D, Helps C, et al. Association between canine leishmaniosis and Ehrlichia canis coinfection: a prospective case-control study. Parasit Vectors. 2018;11:184.

21. Jeronimo SM, Duggal P, Braz RF, Cheng C, Monteiro GR, Nascimento ET, et al. An emerging peri-urban pattern of infection with Leishmania chagasi, the protozoan causing visceral leishmaniasis in northeast Brazil. Scand J Infect Dis. 2004;36:443-9.

22. Lima ID, Queiroz JW, Lacerda HG, Queiroz PV, Pontes NN, Barbosa JD, et al. Leishmania infantum chagasi in northeastern Brazil: asymptomatic infection at the urban perimeter. Am J Trop Med Hyg. 2012;86:99-107.

23. Toepp A, Larson M, Grinnage-Pulley T, Bennett C, Anderson M, Parrish $M$, et al. Safety analysis of Leishmania vaccine used in a randomized canine vaccine/immunotherapy trial. Am J Trop Med Hyg. 2018;98: 1332-8.

24. Larson M, Toepp A, Scott B, Kurtz M, Fowler H, Esfandiari J, et al. Semiquantitative measurement of asymptomatic L. infantum infection and symptomatic visceral leishmaniasis in dogs using Dual-Path Platform(R) CVL. Appl Microbiol Biotechnol. 2017;101:381-90.

25. Toepp A, Larson M, Wilson G, Grinnage-Pulley T, Bennett C, Leal-Lima A, et al. Randomized, controlled, double-blinded field trial to assess Leishmania vaccine effectiveness as immunotherapy for canine leishmaniosis. Vaccine. 2018;36:6433-41.

26. Duthie MS, Lison A, Courtenay O. Advances toward diagnostic tools for managing zoonotic visceral leishmaniasis. Trends Parasitol. 2018. https://doi. org/10.1016/j.pt.2018.07.012.

27. Miro G, Petersen C, Cardoso L, Bourdeau P, Baneth G, Solano-Gallego L, et al. Novel areas for prevention and control of canine leishmaniosis. Trends Parasitol. 2017;33:718-30.

28. Solano-Gallego L, Miro G, Koutinas A, Cardoso L, Pennisi MG, Ferrer L, et al. LeishVet guidelines for the practical management of canine leishmaniosis. Parasit Vectors. 2011:4:86.

29. Alvar J, Gutierrez-Solar B, Molina R, Lopez-Velez R, Garcia-Camacho A, Martinez $P$, et al. Prevalence of Leishmania infection among AIDS patients. Lancet. 1992;339:1427.

30. Esch KJ, Juelsgaard R, Martinez PA, Jones DE, Petersen CA. Programmed death 1-mediated $T$ cell exhaustion during visceral leishmaniasis impairs phagocyte function. J Immunol. 2013;191:5542-50.

31. Cortes S, Vaz Y, Neves R, Maia C, Cardoso L, Campino L. Risk factors for canine leishmaniasis in an endemic Mediterranean region. Vet Parasitol. 2012;189:189-96.

32. Petersen CA, Barr SC. Canine leishmaniasis in North America: emerging or newly recognized? Vet Clin North Am Small Anim Pract. 2009;39:1065-74.

33. Cummings $P$. The relative merits of risk ratios and odds ratios. Arch Pediatr Adolesc Med. 2009;163:438-45.

34. da Silva DA, Madeira Mde F, Abrantes TR, Filho CJ, Figueiredo FB. Assessment of serological tests for the diagnosis of canine visceral leishmaniasis. Vet J. 2013;195:252-3.

35. Kocoglu ME, Tas T, Mengeloglu FZ, Ozsoy S, Bucak O. Evaluation of 4 methods for the serological diagnosis of Epstein-Barr virus infection using an immunofluorescence assay as the reference method. Turk J Med Sci. 2014:44:914-9.

36. Laurenti MD, de Santana Leandro MV Jr, Tomokane TY, De Lucca HR, Aschar M, Souza CS, et al. Comparative evaluation of the DPP((R)) CVL rapid test for canine serodiagnosis in area of visceral leishmaniasis. Vet Parasitol. 2014;205:444-50

37. Monno R, Giannelli G, Rizzo C, De Vito D, Fumarola L. Recombinant K39 immunochromatographic test for diagnosis of human leishmaniasis. Future Microbiol. 2009;4:159-70.
38. Vida B, Toepp A, Schaut RG, Esch KJ, Juelsgaard R, Shimak RM, et al. Immunologic progression of canine leishmaniosis following vertical transmission in United States dogs. Vet Immunol Immunopathol. 2016;169:34-8.

39. Nair AD, Cheng C, Ganta CK, Sanderson MW, Alleman AR, Munderloh UG, et al. Comparative experimental infection study in dogs with Ehrlichia canis, $E$. chaffeensis, Anaplasma platys and A. phagocytophilum. PLoS One. 2016;11: e0148239.

40. Appel MJ, Allan S, Jacobson RH, Lauderdale TL, Chang YF, Shin SJ, et al. Experimental Lyme disease in dogs produces arthritis and persistent infection. J Infect Dis. 1993;167:651-64.

41. Lima WG, Michalick MS, de Melo MN, Luiz Tafuri W, Luiz Tafuri W. Canine visceral leishmaniasis: a histopathological study of lymph nodes. Acta Trop. 2004;92:43-53.

42. Nascimento ET, Moura ML, Queiroz JW, Barroso AW, Araujo AF, Rego EF, et al. The emergence of concurrent HIV-1/AIDS and visceral leishmaniasis in northeast Brazil. Trans R Soc Trop Med Hyg. 2011;105:298-300.

43. Andrade GB, Barreto WT, Santos LL, Ribeiro LR, Macedo GC, Sousa KC, et al. Pathology of dogs in Campo Grande, MS, Brazil, naturally co-infected with Leishmania infantum and Ehrlichia canis. Rev Bras Parasitol Vet. 2014;23:509_ 15.

44. Cota GF, de Sousa MR, de Mendonca AL, Patrocinio A, Assuncao LS, de Faria $\mathrm{SR}$, et al. Leishmania-HIV co-infection: clinical presentation and outcomes in an urban area in Brazil. PLoS Negl Trop Dis. 2014;8:e2816.

45. Belo VS, Struchiner CJ, Barbosa DS, Nascimento BW, Horta MA, da Silva ES, et al. Risk factors for adverse prognosis and death in American visceral leishmaniasis: a meta-analysis. PLoS Negl Trop Dis. 2014;8:e2982.

46. Krause PJ, Telford SR 3rd, Spielman A, Sikand V, Ryan R, Christianson D, et al. Concurrent Lyme disease and babesiosis. Evidence for increased severity and duration of illness. JAMA. 1996;275:1657-60.

47. Belongia EA. Epidemiology and impact of coinfections acquired from Ixodes ticks. Vector Borne Zoonotic Dis. 2002;2:265-73.

48. Azeredo-Coutinho RB, Pimentel MI, Zanini GM, Madeira MF, Cataldo الـ, Schubach $\mathrm{AO}$, et al. Intestinal helminth coinfection is associated with mucosal lesions and poor response to therapy in American tegumentary leishmaniasis. Acta Trop. 2016;154:42-9.

49. Maia Z, Viana V, Muniz E, Goncalves LO, Mendes CM, Mehta SR, et al. Risk factors associated with human visceral leishmaniasis in an urban area of Bahia, Brazil. Vector Borne Zoonotic Dis. 2016;16:368-76.

50. Cardoso L, Yisaschar-Mekuzas Y, Rodrigues FT, Costa A, Machado J, DizLopes D, et al. Canine babesiosis in northern Portugal and molecular characterization of vector-borne co-infections. Parasit Vectors. 2010;3:27.

51. Gaunt S, Beall M, Stillman B, Lorentzen L, Diniz P, Chandrashekar R, et al. Experimental infection and co-infection of dogs with Anaplasma platys and Ehrlichia canis: hematologic, serologic and molecular findings. Parasit Vectors. 2010;3:33.

52. Vrhovec MG, Pantchev N, Failing K, Bauer C, Travers-Martin N, Zahner H. Retrospective analysis of canine vector-borne diseases (CVBD) in Germany with emphasis on the endemicity and risk factors of leishmaniosis. Parasitol Res. 2017;116(Suppl. 1):131-44.

53. Bhadesiya CM, Raval SK. Hematobiochemical changes in ehrlichiosis in dogs of Anand region, Gujarat. Vet World. 2015;8:713-7.

54. Scorza BM, Wacker MA, Messingham K, Kim P, Klingelhutz A, Fairley J, et al. Differential activation of human keratinocytes by Leishmania species causing localized or disseminated disease. J Invest Dermatol. 2017;137: 2149-56.

55. McGill JL, Nair AD, Cheng C, Rusk RA, Jaworski DC, Ganta RR. Vaccination with an attenuated mutant of Ehrlichia chaffeensis induces pathogen-specific CD4+ T cell immunity and protection from ticktransmitted wild-type challenge in the canine host. PLoS One. 2016;11: e0148229.

56. Rudoler N, Harrus S, Martinez-Subiela S, Tvarijonaviciute A, van Straten M, Ceron JJ, et al. Comparison of the acute phase protein and antioxidant responses in dogs vaccinated against canine monocytic ehrlichiosis and naive-challenged dogs. Parasit Vectors. 2015;8:175.

57. Ismail N, Crossley EC, Stevenson HL, Walker DH. Relative importance of Tcell subsets in monocytotropic ehrlichiosis: a novel effector mechanism involved in Ehrlichia-induced immunopathology in murine ehrlichiosis. Infect Immun. 2007:75:4608-20.

58. Baneth G, Harrus S, Gal A, Aroch I. Canine vector-borne co-infections: Ehrlichia canis and Hepatozoon canis in the same host monocytes. Vet Parasitol. 2015;208:30-4. 
59. Kader M, Alaoui-El-Azher M, Vorhauer J, Kode BB, Wells JZ, Stolz D, et al. MyD88-dependent inflammasome activation and autophagy inhibition contributes to Ehrlichia-induced liver injury and toxic shock. PLoS Pathog. 2017:13:e1006644.

60. Travi BL, Tabares CJ, Cadena H, Ferro C, Osorio Y. Canine visceral leishmaniasis in Colombia: relationship between clinical and parasitologic status and infectivity for sand flies. Am J Trop Med Hyg. 2001;64:119-24.

61. Magalhaes-Junior JT, Mota TF, Porfirio-Passos G, Larangeira DF, Franke CR, Barrouin-Melo SM. Xenodiagnosis on dogs with visceral leishmaniasis: canine and sand fly aspects related to the parasite transmission. Vet Parasitol. 2016;223:120-6.

62. Aslan H, Oliveira F, Meneses C, Castrovinci P, Gomes R, Teixeira C, et al. New insights into the transmissibility of Leishmania infantum from dogs to sand flies: experimental vector-transmission reveals persistent parasite depots at bite sites. J Infect Dis. 2016;213:1752-61.

Ready to submit your research? Choose BMC and benefit from:

- fast, convenient online submission

- thorough peer review by experienced researchers in your field

- rapid publication on acceptance

- support for research data, including large and complex data types

- gold Open Access which fosters wider collaboration and increased citations

- maximum visibility for your research: over $100 \mathrm{M}$ website views per year

At BMC, research is always in progress.

Learn more biomedcentral.com/submissions 